\title{
The effect of triiodothyronine on maturation and differentiation of oligodendrocyte progenitor cells during remyelination following induced demyelination in male albino rat
}

\author{
H. El-Tahry ${ }^{a}$, Hany E. Marei ${ }^{b}$, A. Shams ${ }^{a}$, M. El-Shahat ${ }^{a}, *$, H. Abdelaziz ${ }^{c}$, M. Abd El-kader ${ }^{a}$ \\ a Anatomy Department, Faculty of Medicine, Mansoura University, Egypt \\ b Biomedical Research Center, Qatar University, Doha 2713, Qatar University \\ ' Medical Biochemistry Department, Faculty of Medicine, Mansoura University, Egypt
}

\section{A R T I C L E I N F O}

\section{Article history:}

Received 12 October 2015

Received in revised form 1 March 2016

Accepted 1 March 2016

Available online 9 March 2016

\section{Keywords:}

Triiodothyronine

Cuprizone

Remyelination

Oligodendrocyte progenitor cells

\begin{abstract}
A B S T R A C T
Demyelination was induced by two weeks cuprizone treatment. Rats of +ve control and triiodothyronine (T3) then received three subcutaneous injections of either saline or T3 day after day and sacrificed at the end of the third and fifth weeks. Animals in -ve control group received only standard rodent chow. After one week of cuprizone withdrawal the corpus callosum in +ve control and T3 treated rats was still demyelinated as revealed by MBP immunohistochemistry. The assay of PLP gene showed significant increase of T3 treated group compared to both the -ve control and +ve control groups. After three weeks, significant improvement in myelination was detected in T3-treated group compared to +ve control as detected by both MBP immunohistochemistry and electron microscopy. After one week of cuprizone withdrawal, PDGFR $\alpha$ positive cells and gene expression showed significant increase in +ve control and T3-treated groups as compared to -ve control with insignificant difference in between the former two groups. After three weeks of cuprizone withdrawal, PDGFR $\alpha$ positive cells in T3-treated and $+v e$ control groups decreased to the control levels. These results suggest that T3 was effective in improving remyelination when administered during acute phase and might direct progenitor lineage toward oligodendrocytes.
\end{abstract}

(c) 2016 Elsevier Ltd. All rights reserved.

\section{Introduction}

Oligodendrocytes (OLG) provide myelin to axons which is essential for the proper function and maintenance of neurons in the brain. Myelin loss occurs in many demyelinating diseases such as multiple sclerosis (MS) and causes a variety of neurological disabilities in adults (De Groot and Woodroofe, 2001; Lassmann, 2001).

In demyelinating disease lesions, oligodendrocytes are lost either by apoptosis or necrosis (McTigue and Tripathi, 2008) and to promote functional recovery, lesions must be supplied with endogenous oligodendrocyte progenitor cells (OPCs) or with transplanted myelinating cells (Jeffery et al., 1999; Chari and Blakemore, 2002; Blakemore et al., 2002).

\footnotetext{
* Corresponding author.

E-mail address: mshahat50@gmail.com (M. El-Shahat).
}

The adult mammalian subventricular zone (SVZ) of the lateral ventricle contains stem cells that give rise to neurons and glia (Doetsch et al., 1997). It generates the most abundant number of stem cells in the adult brain that are capable of migrating to a long distance (Maki et al., 2013). These endogenous progenitors react to demyelinating lesions and generate remyelinating oligodendrocytes (Nait-Oumesmar et al., 1999).

There is strong evidence supporting that thyroid hormone (TH) acts directly on oligodendrocytes differentiation and maturation processes which has been extensively studied during brain development (Franco et al., 2008). However, TH can also modify the proliferative activity and maturation of OPCs in mature brain (Fernandez et al., 2004) suggesting that OPCs in adult brain are still sensitive to signaling molecules known to regulate oligodendrogenesis during development (Franco et al., 2008).

The present study was designed to clarify the possible effect of T3 on differentiation and maturation of oligodendrocyte precursor cells using oligodendrocyte markers and confirming the result by gene expression. 


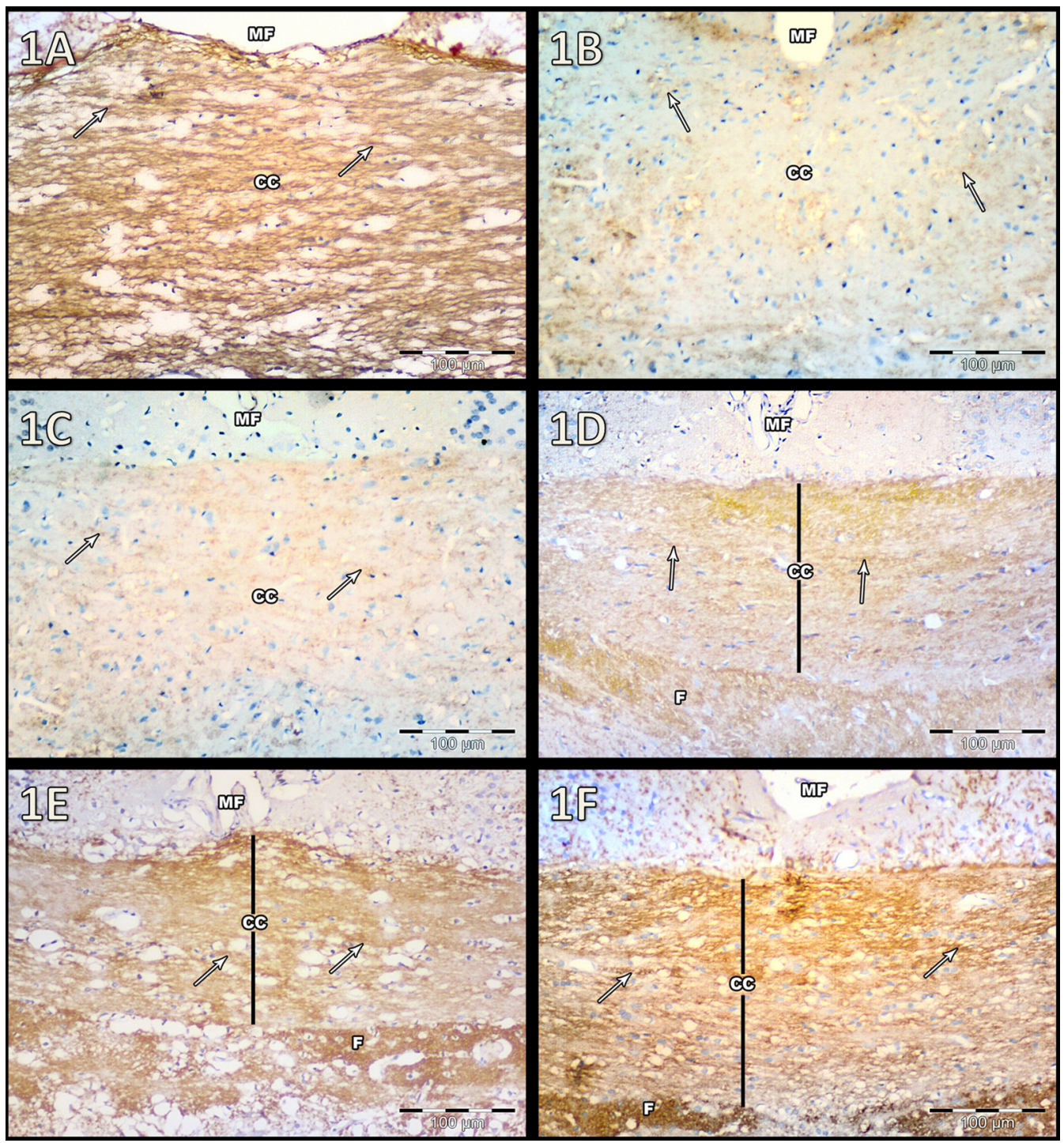

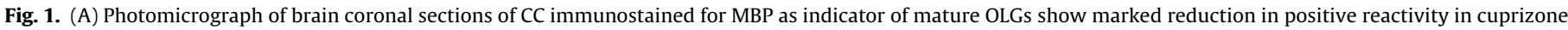

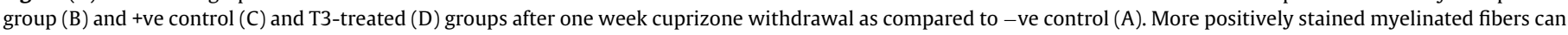

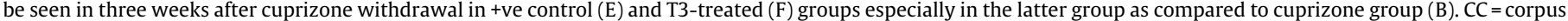
callosum. $\mathrm{MF}=$ median fissure.

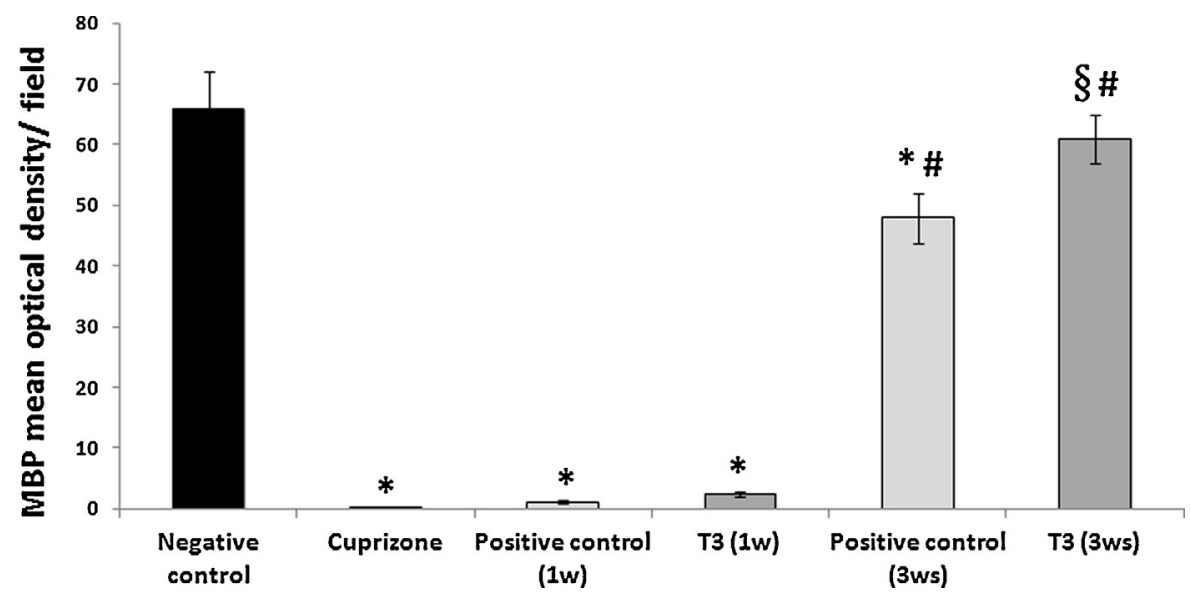

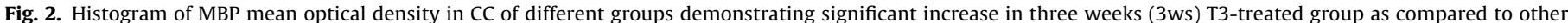

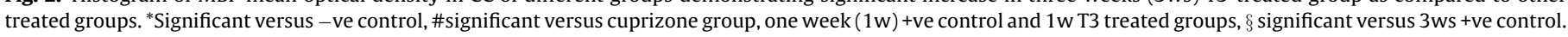
$P<0.001 .1 \mathrm{w}$, one week after cuprizone withdrawal, 3 ws three weeks after cuprizone withdrawal. 


\section{Materials and methods}

\subsection{Animals used}

Sixty six male Sprague Dawley albino rats, weighting 60-70 g and aging three weeks were obtained from Mansoura Experimental Research Center, Egypt. They were housed in stainless steel mesh cages under controlled condition of temperature $\left(23 \pm 3^{\circ} \mathrm{C}\right)$, and relative humidity throughout acclimatization and experimental periods, with ad libitum access to food and water and fixed 12:12-h light/dark cycle. All the experiments were carried out according to the rules and regulations of the Medical Research Ethics Committee of Mansoura Faculty of Medicine.

\subsection{Experimental design}

After an acclimatization period of one week, the rats were randomly divided into four groups: Group I ( - ve control) $(n=24)$ : rats were fed standard rodent chow and divided into three subgroups that were sacrificed at the end of the second week $(n=6)$, third week $(n=12)$ and fifth week $(n=6)$. Group II (cuprizone treated) $(n=6)$ : rats were fed standard rodent chow mixed with cuprizone (Sigma Aldrich) $0.6 \%(w / w)$ for two weeks (Adamo et al., 2006) then sacrificed at the end of the second week. Group III (+ve control) $(n=18)$ : rats were fed standard rodent chow mixed with cuprizone $0.6 \%$ $(\mathrm{w} / \mathrm{w})$ for two weeks then returned to a normal diet. On the third week the rats were subcutaneously injected with saline solution day after day for three injections. Rats in this group were divided into two subgroups sacrificed at the end of the third week $(n=12)$ and the fifth week $(n=6)$. Group IV (triiodothyronine treated $=$ T3 treated) $(n=18)$ : rats were fed on standard rodent chow mixed with cuprizone $0.6 \%(\mathrm{w} / \mathrm{w})$ for 2 weeks then returned to a normal diet with standard rodent chow. On the third week the rats were treated with three subcutaneous injections of triiodothyronine (T3) (Sigma Aldrich) $20 \mu \mathrm{g} / 100 \mathrm{~g}$ body weight day after day (Franco et al., 2008). The animals of this group were divided into two subgroups sacrificed at the end of the third week $(n=12)$ and the fifth week $(n=6)$.

\subsection{Sacrifice of rats and specimens collection}

At the assigned times, rats from each group were anaesthetized with ether inhalation. Rats were fixed by intracardiac perfusion with $4 \%$ paraformaldehyde and $0.1 \%$ glutaraldehyde in $0.05 \mathrm{M}$ phosphate buffer ( $\mathrm{pH} 7.4$ ), decapitated and the brain was removed. Rats used for gene analysis by reverse transcription polymerase chain reaction (RT-PCR) were decapitated and the fresh hemisphere was rapidly removed and preserved in a container containing lysis buffer and was stored in liquid nitrogen at $-80^{\circ} \mathrm{C}$ until analysis.

\subsection{Immunohistochemistry}

Six rats per group were sacrificed after two, three and five weeks and the brain was processed to prepare paraffin sections. Coronal sections were deparaffinized and rehydrated in descending concentrations of ethanol. After routine heat antigen retrieval procedures, sections were treated with $3 \%$ hydrogen peroxide to block endogenous peroxidase and incubated overnight at $4{ }^{\circ} \mathrm{C}$ with a primary antibody raised against myelin basic protein (MBP) (1:100, Sigma Aldrich, Egypt) or platelets derived growth factor receptor alpha $(\operatorname{PDGFR} \alpha)(1: 100$, Abcam, Egypt). After washing with phosphate buffer saline (PBS) (PH7.4), the sections were incubated with secondary antibodies using Mouse and Rabbit Specific HRP/DAB (ABC) Detection IHC Kit (Abcam, Egypt). The cells that displayed brown precipitation were considered to be positive.
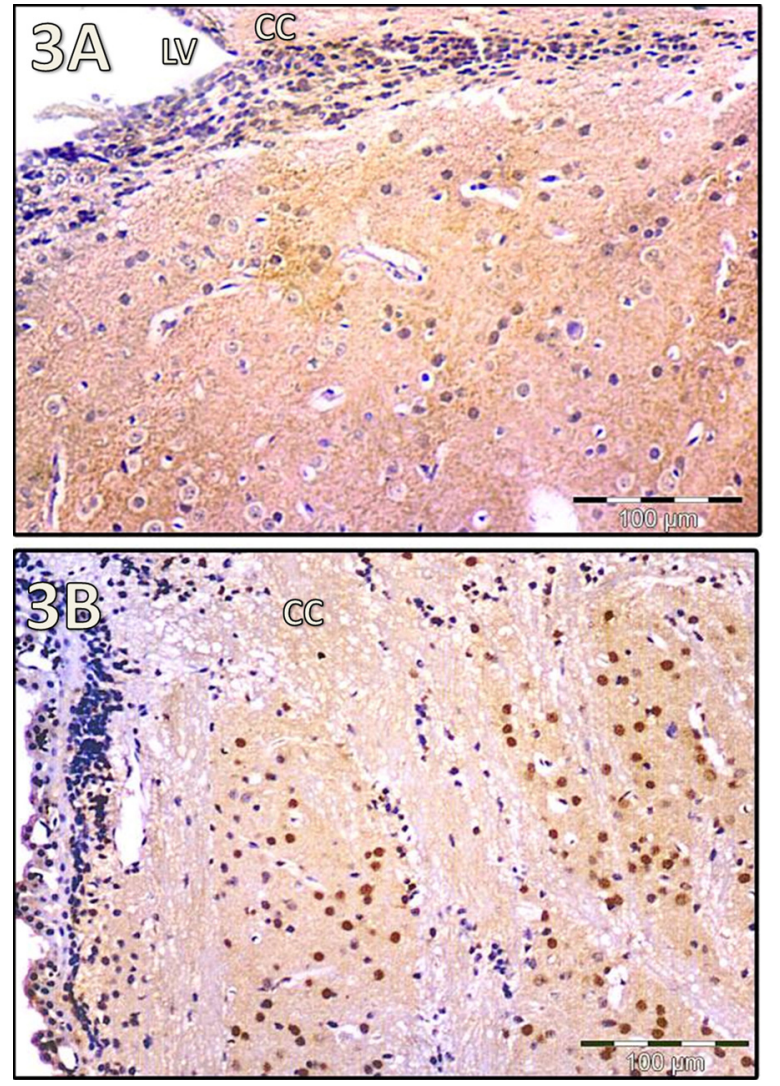

Fig. 3. Few PDGFR $\alpha$ positive cells can be seen peri-ventricularly localized at the dorso-lateral corner of the SVZ in -ve control (A). More dispersed cells can be detected periventricularly, extending laterally and upward beyond the SVZ and in the $\mathrm{CC}$ in cuprizone group (B). CC= corpus callosum. $\mathrm{LV}=$ lateral ventricle.

\subsection{Electron microscopy (EM)}

Six rats per group were taken after two (cuprizone group) and five weeks (from - ve control, +ve control and T3-treated groups, i.e. three weeks after cuprizone withdrawal). The specimens were not taken early after one week of cuprizone withdrawal to ensure evident remyelination. The brain was dissected to obtain the central part of the suprahippocampal portion of the corpus callosum (CC) ( $1 \mathrm{~mm}$ thickness $\times 2 \mathrm{~mm}$ length). The specimens were fixed in $2.5 \%$ gluteraldehyde in phosphate buffer for $24 \mathrm{~h}$, post fixed in osmium tetroxide, dehydrated and embedded in epoxy resin. Ultrathin sections (70-80 nm) were stained with lead citrate and uranyl acetate and examined under Jeol electron microscope (JEM 2100, Japan) at Electron Microscopy Unit, Mansoura University.

\subsection{Gene expression analysis by reverse transcription polymerase chain reaction $(R T-P C R)$}

Six rats per group were taken after three weeks from -ve control, +ve control and T3-treated groups (i.e. one week after cuprizone withdrawal in +ve control and T3-treated). The samples were taken early after demyelination when active cell division and encoding of the syntheses of major myelin proteins are enhanced. Total RNA was extracted from brain samples using RNAspin $^{\mathrm{TM}}$ Total RNA Extraction Kit (Intron Biotechnology). First strand cDNA was synthesized from total RNA templates by The Thermo Scientific $^{\mathrm{TM}}$ RevertAid $^{\mathrm{TM}}$ First Strand cDNA Synthesis Kit (Thermo Scientific, \#K1621). First strand cDNA synthesized with this system could be directly used as a template in PCR.

Polymerase chain reaction (PCR): According to the method of Calza et al. (2002) and Tang et al. (2000), conventional 

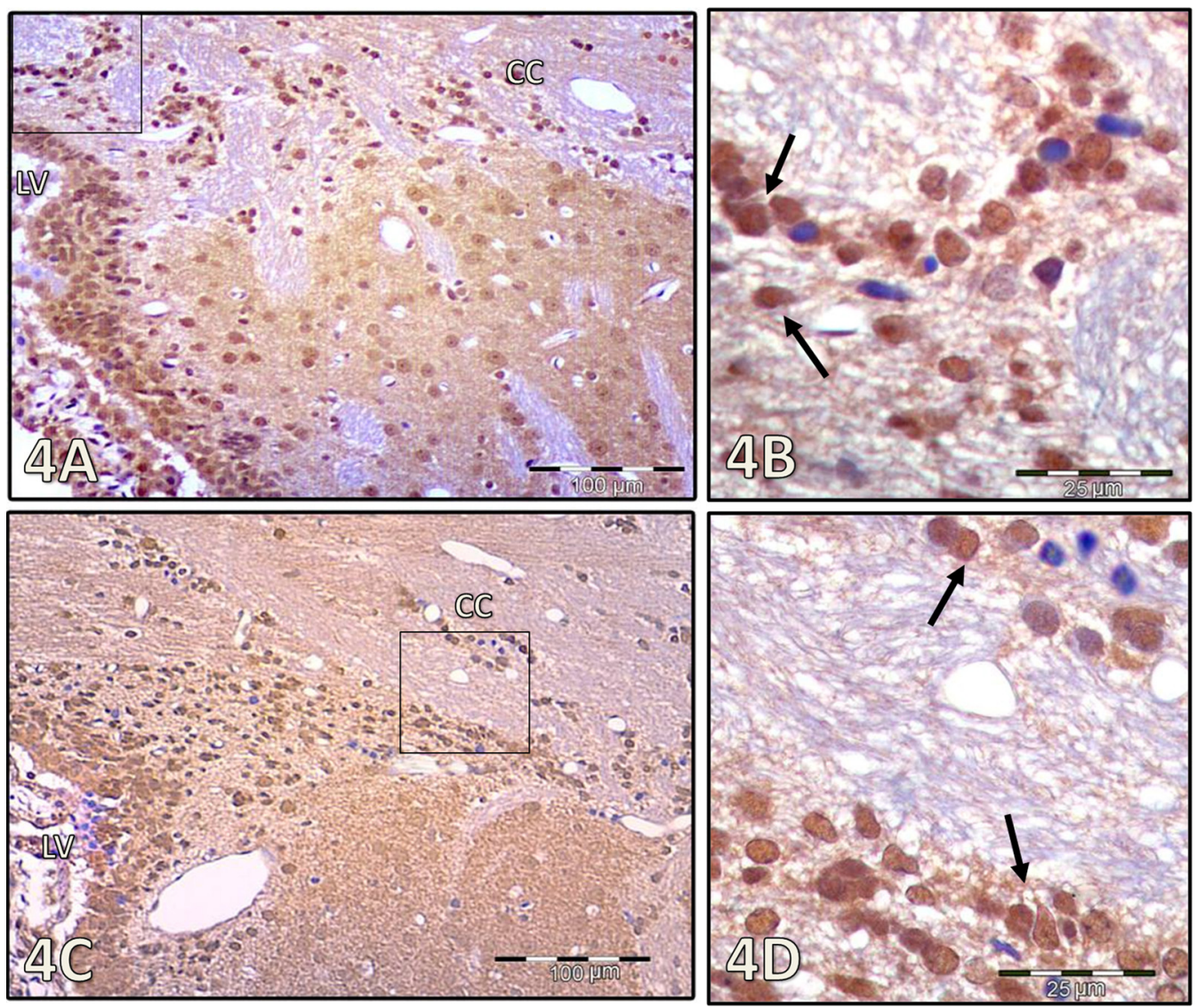

Fig. 4. PDGFR $\alpha$ immunostained cells are more increased after one week in +ve control (A) and T3-treated (B).

method of PCR amplification was done for PDGFR $\alpha$ and proteolipid protein (PLP) genes by using DreamTaq Green PCR Master Mix (2X) (Thermo Sciemtific, \#K1081). GAPDH was used as control gene: GAPDH sense, 5'-TCCATGACAACTTTGGCATCGTGG3'; GAPDH antisense, 5'-GTTGCTGTTGA-AGTCACAGGAGAC-3'. The 2 primers (forward and reverse primers) of each gene were purchased from Biosearch Technologies (USA). PDGFR $\alpha$ sense, $5^{\prime}-$ AGATAGCTT-CATGAGCCGAC-3'; PDGFR $\alpha$ antisense, 5'-GGAACAGGGTCAATGTCTGG-3'; PLP sense, 5'-GTATAGGCAGTCTCTGCGC-3'; PLP antisense, 5'-CAGTATGTCA-TCTATGGAAC-3'. After thawing, $25 \mu \mathrm{L}$ of Green PCR Master Mix (2X) were put in reaction tube with 20 Pico mole of forward primer $(2 \mu \mathrm{L}), 20$ Pico mole of reverse primer $(2 \mu \mathrm{L}), 2 \mu \mathrm{L}$ of template cDNA and $19 \mu \mathrm{L}$ of sterile double distilled water to reach a final volume $50 \mu \mathrm{L}$ and centrifuged briefly. Amplification was performed: for GAPDH by initial denaturation at $95^{\circ} \mathrm{C}$ for $3 \mathrm{~min}$, followed by 25 cycles of $95^{\circ} \mathrm{C}$ for $1 \mathrm{~min}, 60^{\circ} \mathrm{C}$ for $0.5 \mathrm{~min}$ and $72^{\circ} \mathrm{C}$ for $30 \mathrm{~s}$; for PDGFR $\alpha$ by initial denaturation at $95^{\circ} \mathrm{C}$ for $3 \mathrm{~min}$, followed by 30 cycles of $95^{\circ} \mathrm{C}$ for $1 \mathrm{~min}, 60^{\circ} \mathrm{C}$ for $1.5 \mathrm{~min}$ and $72^{\circ} \mathrm{C}$ for $1.5 \mathrm{~min}$; and for PLP by initial denaturation at $95^{\circ} \mathrm{C}$ for $3 \mathrm{~min}$, followed by 30 cycles of $94{ }^{\circ} \mathrm{C}$ for $15 \mathrm{~s}, 53{ }^{\circ} \mathrm{C}$ for $0.5 \mathrm{~min}$ and $72^{\circ} \mathrm{C}$ for $1 \mathrm{~min}$. A final extension was done for all at $72{ }^{\circ} \mathrm{C}$ for $7 \mathrm{~min}$. Products of PCR were subjected to agarose gel electrophoresis using $2 \%$ agarose gel containing $2 \mu \mathrm{L}$ of $10 \mathrm{mg} / \mathrm{ml}$ ethedium bromide (purchased from Sigma-Aldrich), using $1 \times$ Tris-Borate-EDTA Buffer (TBE Buffer) for $1 \mathrm{~h}$ at $70 \mathrm{~V}$.

\subsection{Statistical study}

\subsubsection{Analysis of immunostained sections}

Six tissue sections from each group were prepared and fields from each section were photographed using Olympus ${ }^{\circledR}$ digital cam- era installed on Olympus ${ }^{\circledR}$ light microscope with $1 / 2 \mathrm{X}$ photo adaptor, using $100 \times$ objective (for MBP) and $400 \times$ objective (for PDGFR $\alpha$ ). The result images were analyzed on Intel ${ }^{\circledR}$ Core $I 3{ }^{\circledR}$ based computer using Video Test Morphology ${ }^{\circledR}$ software (Russia) with a specific built-in automated object counting, stain quantification and distance measurement routines. For MBP, area \% of the thresholded stain hue range was calculated. For automated cell counting of PDGFR $\alpha$ stained cells, the image was thresholded using target cell hue range to obtain a binary mask covering the region of interest (ROI). The object counting routine was applied on (ROI) using size range and circularity filter to ignore artifact objects. Results were exported to an excel sheet.

\subsubsection{Analysis of electron microscopic pictures}

Six ultrathin tissue sections from each group were prepared and fields from each section were examined and photographed. For $g$ ratio measurement: images were acquired using a u-tech ${ }^{\circledR}$ frame grabber. The diameter of the axon and the diameter of the same axon with its myelin sheath were measured using the manual line tool. The $g$ ratio was calculated by dividing the obtained diameters. The number of myelinated axons per field was counted manually. Results were exported to an excel sheet.

\subsubsection{Quantification of PCR product}

Fluorescent bands were detected on agarose gel in six samples from each group and photographed by digital camera. Gel images were digitized using Viewpix 700 gel scanner using transparency unit, gel images were saved in TIFF format. Images were analyzed using total lab quant software to obtain band intensity represented as area under peak. 


\subsection{Statistical analysis}

All data were presented as mean \pm SD using the computer program SPSS version 17.0. The significance of difference between groups was tested using ANOVA (analysis of variance) followed by post hoc tukey for multiple comparisons. A $P$ value $<0.05$ was considered statistically significant. And a $P$ value $<0.001$ was considered highly significant in all analyses.

\section{Results}

\subsection{Immunohistochemistry for $M B P$}

-ve control group. The corpus callosum (CC) of the control group was completely myelinated as shown with MBP immunohistochemistry (Fig. 1A).

Cuprizone treated group. After two weeks of cuprizone intoxication callosal fibers were nearly completely demyelinated in the central area. The peripheral areas of CC revealed only sparse myelinated fragments (Fig. 1B).

+ve control group. After one week of cuprizone withdrawal, CC was still demyelinated with rare and extremely scattered myelinated fragments (Fig. 1C). After three weeks of cuprizone withdrawal moderately myelinated fibers appeared dispersed in central and peripheral parts of CC (Fig. 1E).

Triiodothyronine (T3) treated group. After one week of cuprizone withdrawal the corpus callosum in T3-treated rats was still demyelinated with scattered myelinated fragments approaching the central area of CC (Fig. 1D). While after three weeks, myelinated fibers appeared more densely spreading in central and peripheral parts of CC (Fig. 1F).

In all experimental groups, the thickness of CC was still decreased compared to (-ve) control group. The optical color density of the corpus callosum stained with MBP immunohistochemistry for the different groups is presented in (Fig. 2). In +ve control and T3 treatment for one week, the color density was not significantly increased as compared to cuprizone group. After three weeks of cuprizone withdrawal, +ve control group showed significant increase as compared to cuprizone group but still significantly lower than $(-\mathrm{ve})$ control. On the other hand, the color density of T3 treated group showed significant increase than all other treated groups and was comparable to (-ve) control.

\subsection{Immunohistochemistry for $(P D G F R \alpha)$}

The expression of PDGFR $\alpha$ was studied in the CC, the subventricular zone and the surrounding areas to clarify the number, site and migration of oligodendrocyte progenitor cells (OPCs) in different groups.

- ve control group showed weak positive (PDGFR $\alpha$ ) immunoreactivity. The positive cells were detected periventricularly and localized at the dorso-lateral corner of the SVZ (Fig. 3A).

Cuprizone treated group. The positive cells showed significant increase compared to -ve control group. They were more dispersed and detected periventricularly, extending laterally and upward beyond the SVZ suggesting migration away from the SVZ. Also, some cells could be detected inside the $\mathrm{CC}$ which may indicate migration of OPCs toward the lesion (Figs. 3B and 6).

+ve control group. After one week, the positive cells showed significant increase compared to -ve control and were detected periventricularly especially at the dorso-lateral corner of the SVZ and extending upward beyond the SVZ. Positive cells appeared more inside the corpus callosum suggesting its dorsal migration (Figs. 4A, B, 6). After three weeks, the positive cells were not significantly higher than -ve control. However, it showed significant

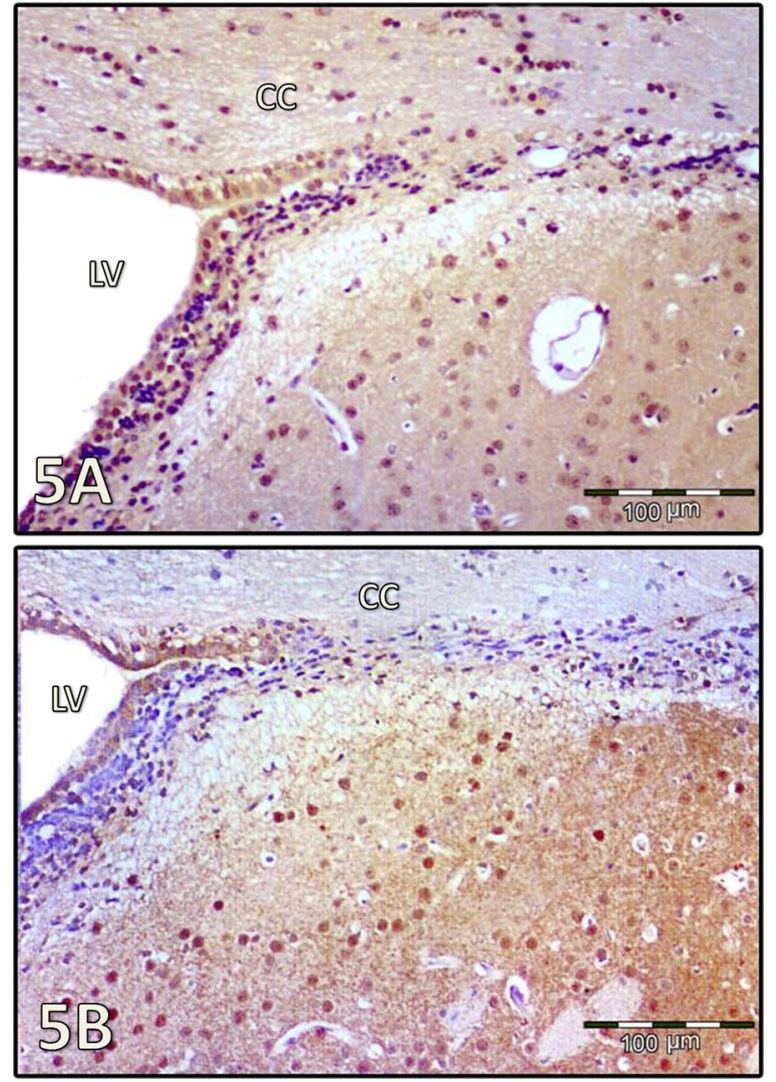

Fig. 5. Reduction in the number of PDGFR $\alpha$ positive cells is observed after three weeks of cuprizone withdrawal in +ve control (A) and especially in T3-treated group (B).

decrease compared to cuprizone group and one week +ve control group. They were detected periventricularly and still present inside the corpus callosum (Figs. 5A and 6).

T3 treated group. After one week, the positive cells were not significantly increased compared to one week +ve control group with also a similar distribution (Figs. 4C, 4D, 6). After three weeks, the positive cells were detected periventricularly. The number of positive cells were decreased to -ve control level (Figs. 5B, 6).

\subsection{Electron microscopy}

-ve control group. Nearly all axons of CC were myelinated with different diameters and contained round or oval mitochondria (Fig. 7A). The number of myelinated axons per field was $379.67 \pm 33.52$ (Fig. 8A). The average $g$ ratio was $0.61 \pm 0.07$ (Fig. 8B). Oligodendrocytes had oval dark nuclei with coarse chromatin some of it was adherent to nuclear membrane and dark cytoplasm containing round or oval mitochondria (Fig. 7A).

Cuprizone treated group. The axons were mostly demyelinated or had very thin, irregular with blebbing myelin sheath. Evidence of axon degeneration as presence of demyelinated swollen axons with distorted cytoskeleton, myelin sheath vacuolation and megamitochondria were observed (Fig. 7B). The number of myelinated axons was significantly decreased as compared to the -ve control group (Fig. 8A). The average $\mathrm{g}$ ratio was significantly increased as compared to -ve control group (Fig. 8B). Oligodendrocytes in CC of demyelinated group revealed irregular nuclei with dense chromatin. The cytoplasm showed different electron dense materials and megamitochondria. Some oligodendrocytes showed signs of apoptosis in the form of irregular condensed chromatin (pyknotic nuclei) (Fig. 7B). 


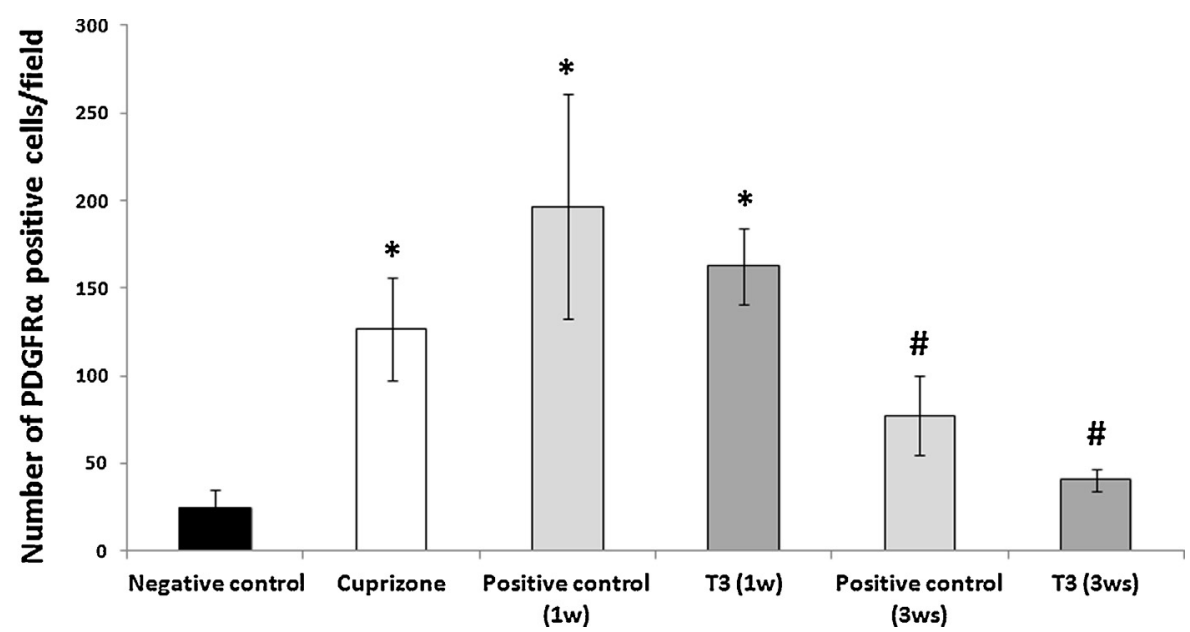

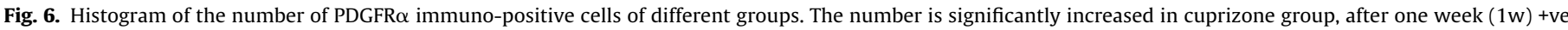

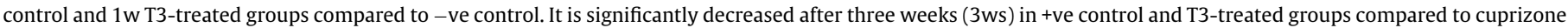

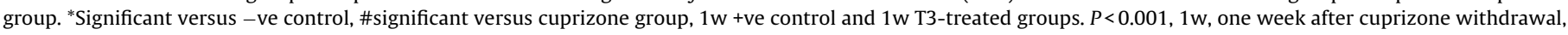
3 ws three weeks after cuprizone withdrawal.
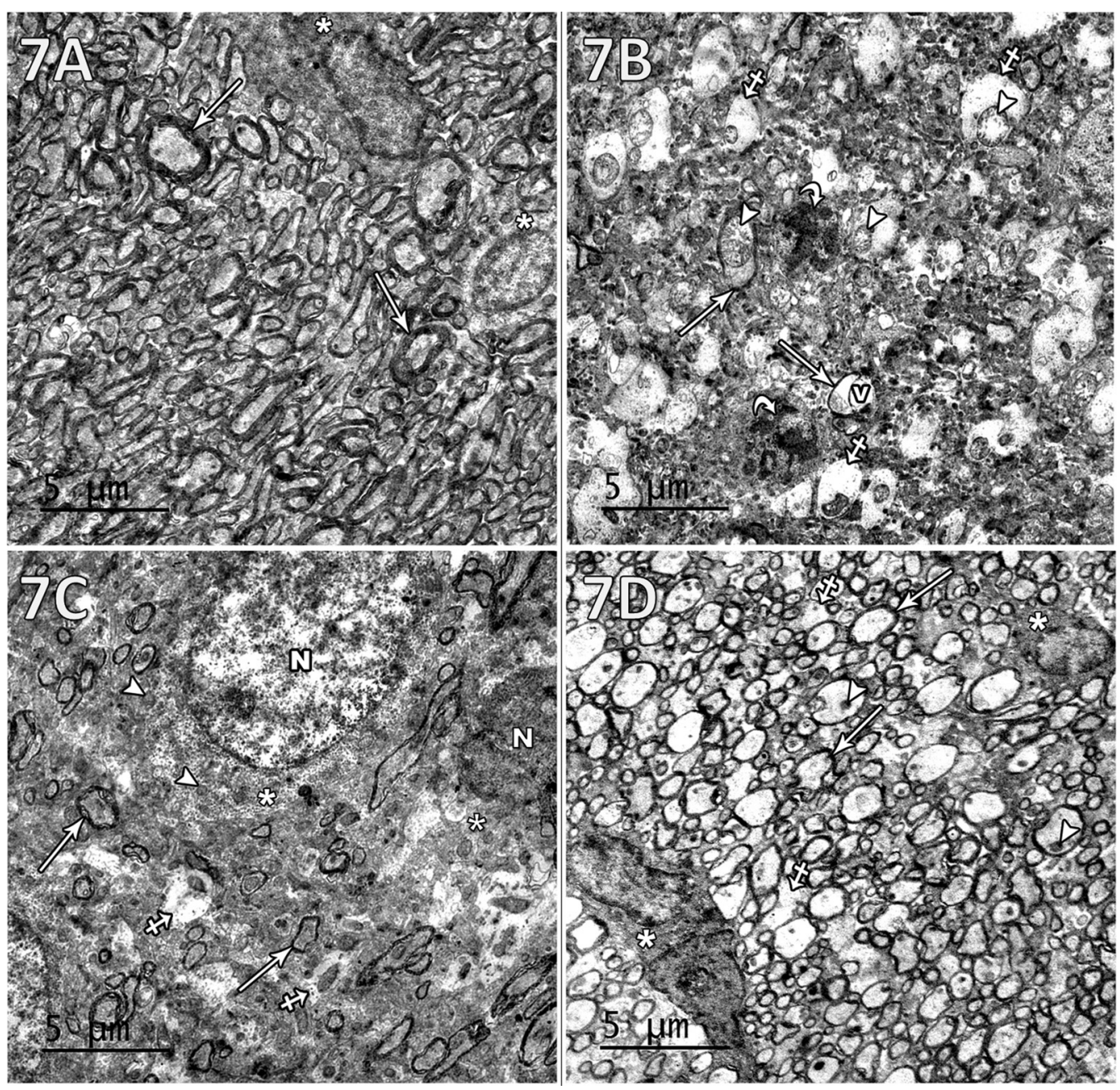

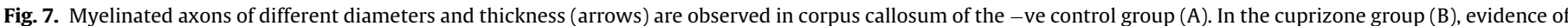

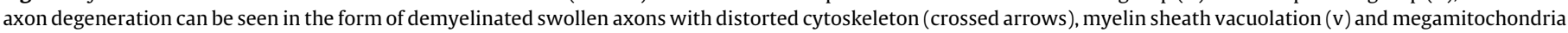

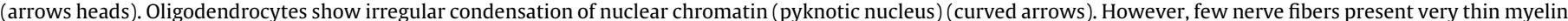

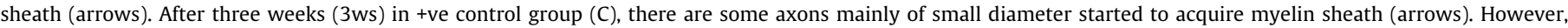

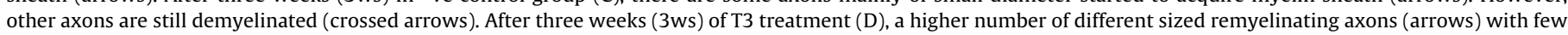
demyelinating axons (crossed arrows) are observed. $\left({ }^{*}\right)=$ oligodendrocyte. $\mathrm{V}=$ vacuolation. $\mathrm{N}=$ nucleus. 
+ve control group. After three weeks of cuprizone withdrawal, some axons mainly of small diameter started to acquire thin myelin sheath. However, other axons were still demyelinated (Fig. 7C). The number of myelinated axons was although significantly higher than the cuprizone group, it was still significantly lower than the -ve control group (Fig. 8A). The average g ratio was significantly lower than the cuprizone group but still significantly higher than the -ve control group (Fig. 8B). Oligodendrocytes in CC of three weeks +ve control group revealed oval dark nuclei with coarse chromatin some of it was adherent to nuclear membrane and less dark cytoplasm (young oligodendrocyte) containing average sized mitochondria (Fig. 7C).

T3 treated group. There were many axons acquired myelin sheath with variable thickness that appeared thicker than that of +ve control group (Fig. 7D). The number of myelinated axons was significantly higher than both the cuprizone group and three weeks +ve control group. There was no significant difference between this group and the -ve control group (Fig. 8A). The g ratio was not significantly different from three weeks +ve control group (Fig. 8B). Oligodendrocytes had oval dark nuclei with coarse chromatin some of it was adherent to nuclear membrane and darker cytoplasm (more mature oligodendrocyte) containing average sized mitochondria (Fig. 7D).

\subsection{Gene expression (RT-PCR)}

\subsubsection{Expression of GAPDH as a control gene}

In reverse transcription polymerase chain reaction (RT-PCR) the GAPDH appeared at $375 \mathrm{bp}$. The difference in the expression of GAPDH after one week between the -ve control, +ve control and T3 treated groups was not significant indicating valid mRNA in all specimens (Fig. 9A).

\subsubsection{Expression of PDGFR $\alpha$ as a marker for OPCS}

In RT-PCR, the PDGFR $\alpha$ appeared at $1016 \mathrm{bp}$. The assay of PDGFR $\alpha$ revealed significant increase after one week in both +ve control and T3 treated groups compared to the -ve control group. However, the difference between one week +ve control and T3 treated groups was insignificant (Fig. 9B).

\subsubsection{Expression of PLP as a marker for mature OLGs}

In RT-PCR, the PLP appeared at $357 \mathrm{bp}$. The assay of PLP revealed significant increase in one week $\mathrm{T} 3$ treated group compared to both the -ve control and one week +ve control groups (Fig. 9C).

\subsubsection{Summary of results}

The results of the present work are summarized in Fig. 10 showing significant enhancement of myelination in corpus callosum of T3 treated animals compared to +ve control which was apparent after three weeks of cuprizone withdrawal.

\section{Discussion}

In demyelinating diseases, oligodenrocytes which provide myelin sheath to axons become lost (Lassmann, 2001). In the present work and in agreement with Adamo et al. (2006), two weeks of cuprizone treatment resulted in very weak immunoreactivity for the mature OLG marker MBP in the CC as compared to the control group indicating damage of oligodendrocytes by the toxic effect of cuprizone.

In +ve control, after one week of cuprizone withdrawal, the saline treated animals displayed few cells expressing MBP in the CC indicating that they were at early stages of spontaneous remyelination. Matsushima and Morell (2001), Crawford et al. (2009) and Steelman et al. (2012) also reported that the cuprizone demyelination stops upon termination of cuprizone administration and
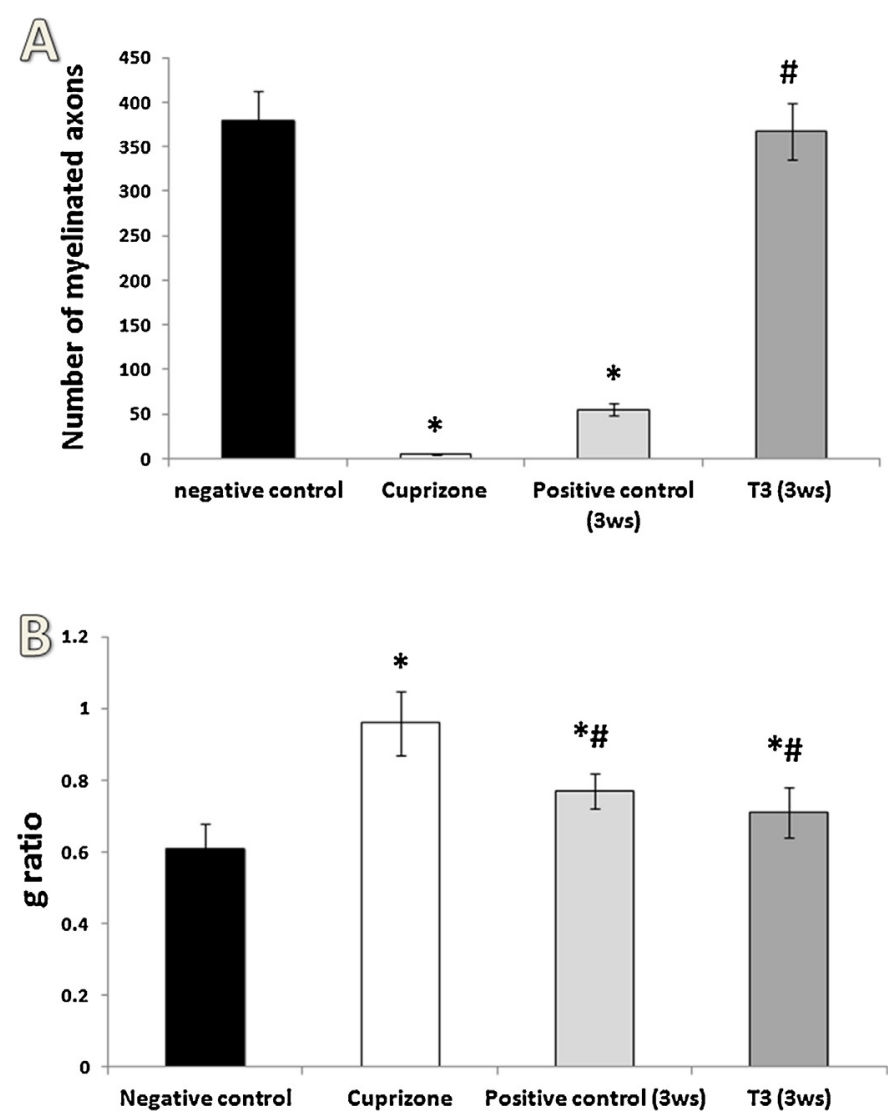

Fig. 8. Histograms of the number of myelinated axons (A) and g ratio (B). The number of myelinated axons is significantly higher after three weeks (3ws) in T3-treated group compared to cuprizone group and $3 w s+v e$ control. The g ratio is significantly lower in 3ws +ve control and 3ws T3-treated groups compared to cuprizone group, but still significantly higher than -ve control. *significant versus -ve control, \#significant versus cuprizone group. $P<0.05,1 \mathrm{w}$, one week after cuprizone withdrawal, 3 ws three weeks after cuprizone withdrawal.

is followed by spontaneous remyelination. However, Franco et al. (2008) observed that maximum demyelination was detected histologically one week after cuprizone withdrawal with significant decrease in MBP expression.

In T3 treated group, treatment for one week with T3 after cuprizone withdrawal induced significant expression of PLP gene as revealed by RT-PCR compared to both the -ve control and +ve control groups. PLP gene encodes the myelin protein PLP indicating increased rate of differentiation of OPCs into mature OLGs induced by treatment of T3 in acute phase of demyelination. However, myelin formation as indicated by MBP immunonohistochemistry was not significantly increased in T3 treated group after one week of cuprizone withdrawal to the contrary of Franco et al. (2008) who reported that T3 treatment induced significant increase of MBP immunohistochemistry after one week of cuprizone withdrawal. In this study, MBP immunonohistochemistry became significantly higher in T3 treated group compared to animals recovering spontaneously after three weeks of T3 treatment indicating enhanced remyelination.

In the SVZ, OPCs are induced to proliferate, migrate toward demyelinated areas and differentiate into mature myelinating oligodendrocytes (Picard-Riera et al., 2002). In the present work, PDGFR $\alpha$ (a marker of OPCs) immunohistochemical stain showed increased expression in cuprizone group than -ve control group. In cuprizone group, the PDGFR $\alpha$ immunopositive cells were scattered lateral to the wall of the lateral ventricle while in the -ve control group, the PDGFR $\alpha$ immunopositive cells were localized 

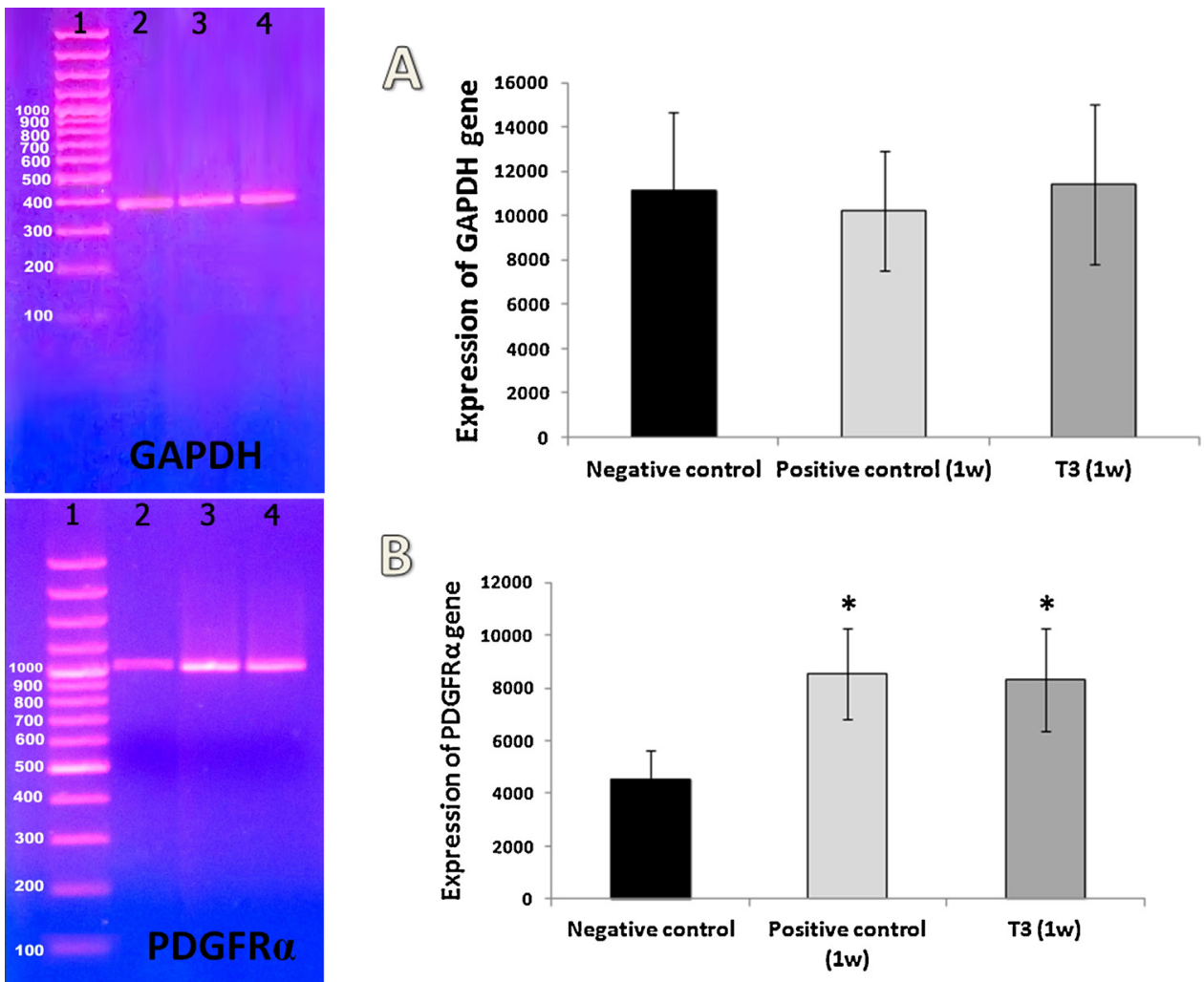

D)
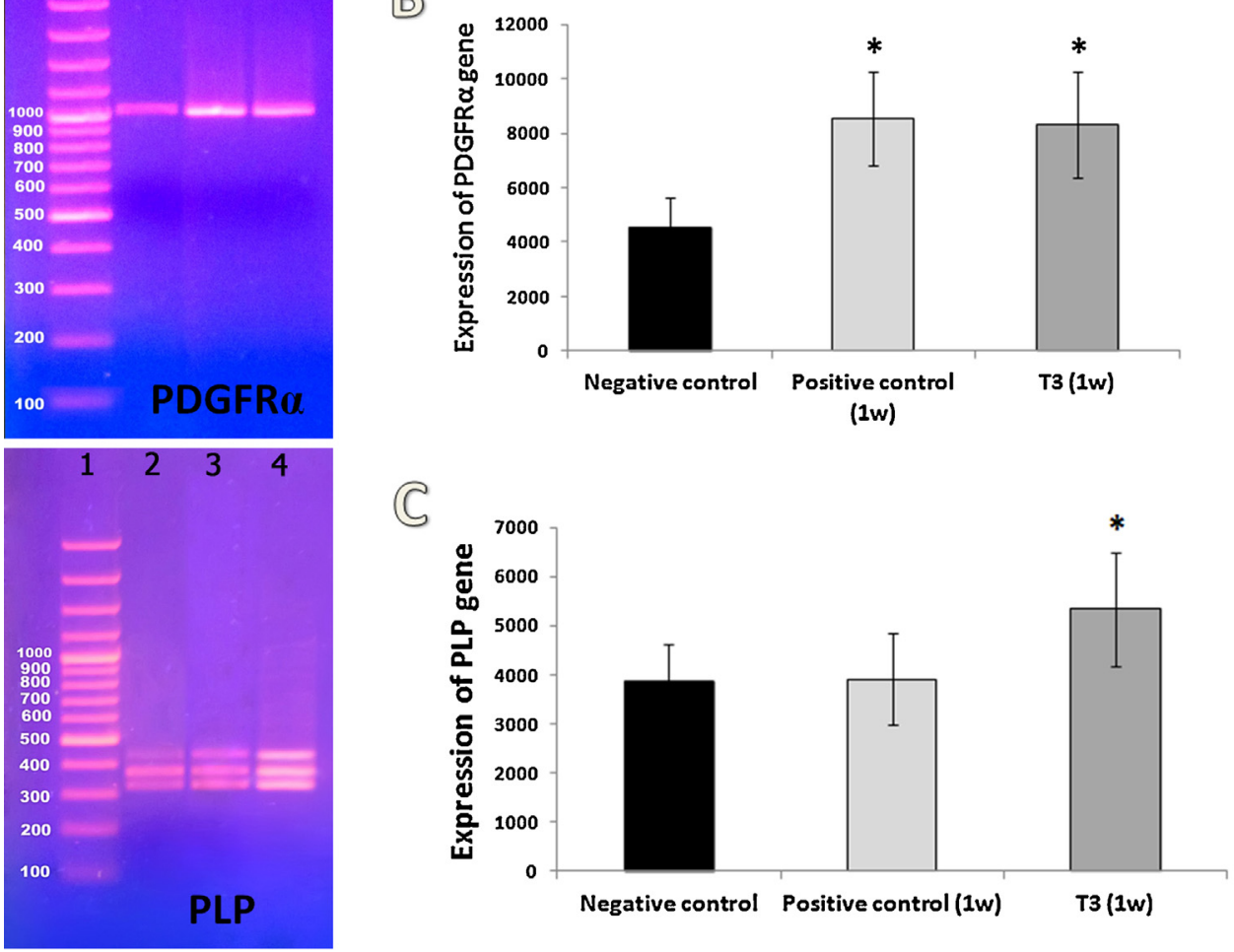

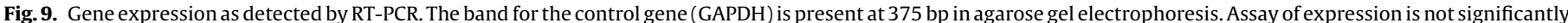

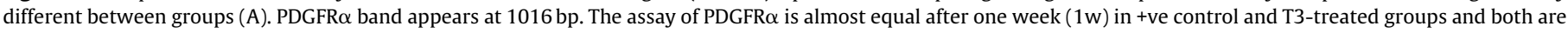

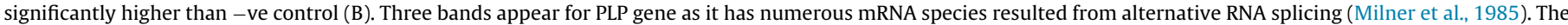

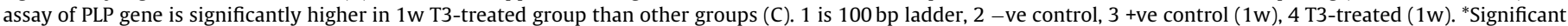
versus - ve control in (B), *significant versus - ve control and +ve control in (C). $P<0.05$.

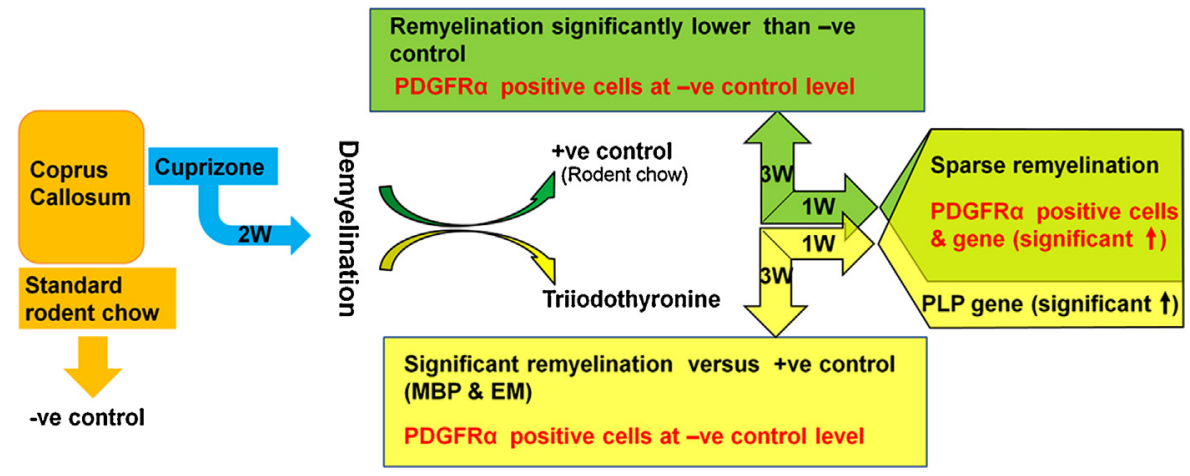

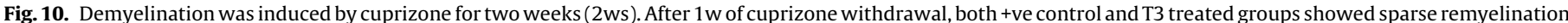

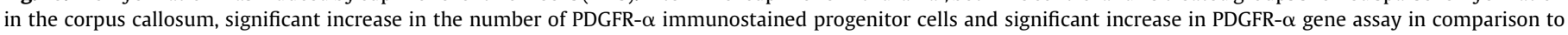

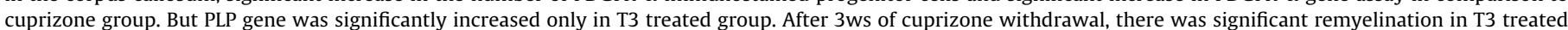

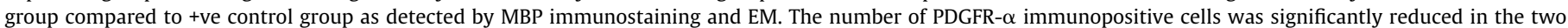
groups compared to cuprizone group but without significant difference in between them. 
at the dorsolateral aspect of the lateral ventricle in consistence with the result of Adamo et al. (2006) using NG2 as a marker of OPCs and Franco et al. (2008) using the neuroepithelial stem cell marker Nestin. The increased and scattered aspects of PDGFR $\alpha$ immunopositive cells can indicate proliferation and possible migration of OPCs which was induced by the lesion. Tsiperson et al. (2015) also demonstrated that following demyelination by cuprizone, an increase in PDGFR $\alpha$ progenitors was observed in wild mice as a result of increased DNA synthesis and cell proliferation. According to Decker et al. (2002), cytokines and growth factors released either from widespread inflammatory and glial cells (Levine, 1994) or from damaged axons after demyelination (Di Bello et al., 1999), induce OPCs to reenter the cell cycle after demyelination.

After one week of cuprizone withdrawal from the diet, PDGFR $\alpha$ immunopositive cells were still significantly increased in both +ve control rats and T3-treated rats in comparison to -ve control, without a significant change compared to cuprizone group. Also, there was no significant difference between +ve control or T3 treated animals. The PDGFR $\alpha$ immunopositive cells in both groups were more dispersed extending upward beyond the SVZ and appeared more inside the corpus callosum suggesting its migration toward demyelinated areas. Supporting this view is the work of Nait-Oumesmar et al. (1999) who described ${ }^{3} \mathrm{H}$-thymidine labeled oligodendrocyte and astrocyte progenitor cells to migrate from SVZ to demyelination lesion induced by lysolecithin. However, Franco et al. (2008) demonstrated that the density of OPCs was markedly reduced after $\mathrm{T} 3$ treatment and became concentrated as a thin rim located nearer to the ventricular wall. They explained that $\mathrm{T} 3$ treatment accelerates the remyelination by increase the differentiation of these progenitor cells to mature OLGs.

This study showed that the gene expression of PDGFR $\alpha$ revealed by RT-PCR was significantly increased after one week in both +ve control and T3 treated groups compared to the -ve control group. In support of the immunohistochemical results, no significant difference was found between the two experimental groups. However, Fernandez et al. (2004) found that PDGFR $\alpha$ gene expression increased significantly after T4 in treated group than non treated group suggesting that a larger number of OPCs are formed in treated rats. This may return to the different experimental model as their study was done in an autoimmune inflammatory demyelination model in which the OPCs may be targeted.

Three weeks after cuprizone withdrawal, there was a significant decrease in the number of OPCs (PDGFR $\alpha$ immunopositive) in +ve control and T3 treated animals compared to cuprizone group and +ve control after one week without significant increase above the -ve control level. This was accompanied by a significant increase in the MBP +ve immunoreactivity in T3 treated animals which supports the favorable effects of $\mathrm{TH}$ on remyelination suggesting that more immature cells were converted into mature OLGs.

Evidence of axon degeneration was observed in the $\mathrm{CC}$ of cuprizone group by EM as the presence of demyelinated swollen axons containing distorted cytoskeleton, myelin sheath vacuolation, megamitochondria and apoptotic cells. These results are in consistence with that of Irvine and Blakemore (2006) and Steelman et al. (2012). However, Silvestroff et al. (2012) using the same intoxication protocol in rats, showed that CC was not severely affected after two weeks of cuprizone ingestion and severe demyelination in the CC appeared one week later. This apparent discrepancy could be explained by the brain sections analyzed in each study. In the present study, the supra-hippocampal portion of the CC which at more caudal position was described, while in the study of Silvestroff et al. (2012) the brain sections were analyzed at a more rostral level. In fact, it has been shown that cuprizone induced demyelination produces a dramatic rostrocaudal gradient of white matter demyelination, with the caudal CC more injured than rostral area (Wu et al., 2008; Xie et al., 2010).
In the present study, three weeks after cuprizone withdrawal in +ve control group, some axons in CC mainly of small diameter started to acquire myelin sheath. However, other axons were still demyelinated. The $g$ ratio of newly myelinated axon, although significantly lower than the demyelinated group, was still significantly higher than the -ve control group indicating a thinner newly formed myelin.

After three weeks of cuprizone withdrawal the number of myelinated axons in T3 treated group approximated the -ve control level and was significantly higher than three weeks +ve control group. However, the newly formed myelin was thinner than that of the -ve control group. In agreement with Silvestroff et al. (2012) the ultrastructure investigation of the present work suggests that $\mathrm{TH}$ enhances the remyelination by increasing the number of remyelinated axons.

TH plays an important part in regulating oligodendrocyte lineage and maturation in vivo (Baas et al., 2002). At first, OPCs divide for a defined number of cycles over a defined time interval which depends on PDGF and involves the cyclin-dependent protein kinase inhibitor p27_Kip (Durand et al., 1998). Then, TH stops cell division and initiates differentiation which involves p53 family proteins (Tokumoto et al., 2001). Franco et al. (2008) suggested that during the initial stages, demyelination activates thyroid hormone receptor $\alpha(\mathrm{TR} \alpha)$ in the SVZ and makes undifferentiated proliferating cells responsive to T3. Exposure to T3 at this time promotes OLG differentiation and enhances myelination through TR $\beta$.

\section{Conclusions}

In the present study, it was observed that T3 was effective in improving remyelination when administered early during intense proliferation and migration of OPCs hence stimulated lineage toward oligodendrocytes as indicated by the increased expression of PLP and MBP and number of myelinated axons in EM. This was especially evident after three weeks of cuprizone withdrawal.

\section{References}

Adamo, A.M., Paez, P.M., Escobar Cabrera, O.E., Wolfson, M., Franco, P.G., Pasquini, J.M., et al., 2006. Remyelination after cuprizone-induced demyelination in the rat is stimulated by apotransferrin. Exp. Neurol. 198, 519-529.

Baas, D., Legrand, C., Samarut, J., Flamant, F., 2002. Persistence of oligodendrocyte precursor cells and altered myelination in optic nerve associated to retina degeneration in mice devoid of all thyroid hormone receptors. Proc. Natl. Acad. Sci. U. S. A. 99, 2907-2911.

Blakemore, W.F., Chari, D.M., Gilson, J.M., Crang, A.J., 2002. Modelling large areas of demyelination in the rat reveals the potential and possible limitations of transplanted glial cells for remyelination in the CNS. Glia 38, 155-168.

Calza, L., Fernandez, M., Giuliani, A., Aloe, L., Giardino, L., 2002. Thyroid hormone activates oligodendrocyte precursor and increases a myelin-forming protein and NGF content in the spinal cord during experimental allergic encephalomyelitis. Proc. Natl. Acad. Sci. U. S. A. 99, 3258-3263.

Chari, D.M., Blakemore, W.F., 2002. Efficient recolonisation of progenitor-depleted areas of the CNS by adult oligodendrocyte progenitor cells. Glia 37, 307-313.

Crawford, D.K., Mangiardi, M., Xia, X., Lopez-Valdes, H.E., Tiwari-Woodruff, S.K., 2009. Functional recovery of callosal axons following demyelination: a critical window. Neuroscience 164, 1407-1421.

De Groot, C.J., Woodroofe, M.N., 2001. The role of chemokines and chemokine receptors in CNS inflammation. Prog. Brain Res. 132, 533-544.

Decker, L., Picard-Riera, N., Lachapelle, F., Baron-Van Evercooren, A., 2002. growth factor treatment promotes mobilization of young but not aged adult subventricular zone precursors in response to demyelination. J. Neurosci. Res. 69, 763-771.

Di Bello, I.C., Dawson, M.R.L., Levine, J.M., Reynolds, R., 1999. Generation of oligodendroglial progenitors in acute inflammatory demyelinating lesions of the rat brain stem is associated with demyelination rather than inflammation. J. Neurocytol. 28, 365-381.

Doetsch, F., Garcia-Verdugo, J.M., Alvarez-Buylla, A., 1997. Cellular composition and three-dimensional organization of the subventricular germinal zone in the adult mammalian brain. J. Neurosci. 17, 5046-5061.

Durand, B., Fero, M.L., Roberts, J.M., Raff, M., 1998. p27/Kip1 alters the response of cells to mitogen and is part of a cell intrinsic timer that arrests the cell cycle and initiates differentiation. Curr. Biol. 8, 431-440. 
Fernandez, M., Pirondi, S., Manservigi, M., Giardino, L., Calza‘, L., 2004. Thyroid hormone participates in the regulation of neural stem cells and oligodendrocyte precursor cells in the central nervous system of adult rat. Eur. J. Neurosci. 20, 2059-2070.

Franco, P., Silvestroff, L., Soto, E., Pasquini, J., 2008. Thyroid hormones promote differentiation of oligodendrocyte progenitor cells and improve remyelination after cuprizone-induced demyelination. Exp. Neurol. 212, 458-467.

Irvine, K.-A., Blakemore, W.F., 2006. Age increases axon loss associated with primary demyelination in cuprizone-induced demyelination in C57BL/6 mice. J. Neuroimmunol. 175, 69-76.

Jeffery, N.D., Crang, A.J., O‘leary, M.T., Hodge, S.J., Blakemore, W.F., 1999. Behavioural consequences of oligodendrocyte progenitor cell transplantation into experimental demyelinating lesions in the rat spinal cord. Eur. J. Neurosci. $11,1508-1514$.

Lassmann, H., 2001. Classification of demyelinating diseases at the interface between etiology and pathogenesis. Curr. Opin. Neurol. 14, 253-258.

Levine, J.M., 1994. Increased expression of the NG2 chondroitin-sulfate proteoglycan after brain injury. J. Neurosci. 14, 4716-4730.

Maki, T., Liang, A.C., Miyamoto, N., Lo, E.H., Arai, K., 2013. Mechanisms of oligodendrocyte regeneration from ventricular-subventricular zone-derived progenitor cells in white matter diseases. Front. Cell. Neurosci. 7, 275.

Matsushima, G.K., Morell, P., 2001. The neurotoxicant, cuprizone, as a model to study demyelination and remyelination in the central nervous system. Brain Pathol. 11, 107-116.

McTigue, D.M., Tripathi, R.B., 2008. The life, death and replacement of oligodendrocytes in the adult CNS. J. Neurochem. 107, 1-19.

Milner, R.J., Lai, C., Nave, K.A., Lenoir, D., Ogata, J., Sutcliffe, J.G., 1985. Nulceotide sequence of two mRNAs for rat myelin proteolipid protein. Cell 42, 931-939.

Nait-Oumesmar, B., Decker, L., Lachapelle, F., Avellana-Adalid, V., Bachelin, C., Van Evercooren, A.B., 1999. Progenitor cells of the adult mouse subventricular zone proliferate, migrate and differentiate into oligodendrocytes after demyelination. Eur. J. Neurosci. 11, 4357-4366.

Picard-Riera, N., Decker, L., Delarasse, C., Goude, K., Nait-Oumesmar, B., Liblau, R., et al., 2002. Experimental autoimmune encephalomyelitis mobilizes neural progenitors from the subventricular zone to undergo oligodendrogenesis in adult mice. Proc. Natl. Acad. Sci. U. S. A. 99, 13211-13216.

Silvestroff, L., Bartucci, S., Pasquini, J., Franco, P., 2012. Cuprizone induced demyelination in the rat cerebral cortex and thyroid hormone effects on cortical remyelination. Exp. Neurol. 235, 357-367.

Steelman, A.J., Thompson, J.P., Li, J., 2012. Demyelination and remyelination in anatomically distinct regions of the corpus callosum following cuprizone intoxication. J. Neurosci. Res. 72 (1), 32-42.

Tang, D.G., Tokumoto, Y.M., Raff, M.C., 2000. Long-term culture of purified postnatal oligodendrocyte precursor cells: evidence for an intrinsic maturation program that plays out over months. J. Cell Biol. 148, 971-984.

Tsiperson, V., Huang, Y., Bagayogo, I., Song, Y., VonDran, M.W., DiCicco-Bloom, E., Dreyfus, C.F., 2015. Brain-derived neurotrophic factor deficiency restricts proliferation of oligodendrocyte progenitors following cuprizone-induced demyelination. ASN Neuro 7 (1), 1-11.

Tokumoto, Y.M., Tang, S.G., Raff, M.C., 2001. Two molecularly distinct intracellular pathways to oligodendrocyte differentiation: role of a p53 family protein. EMBO J. 20, 5261-5268.

Wu, Q., Yang, Q., Cate, H., Kemper, D., Binder, M., Wang, H., et al., 2008. MRI identification of the rostral-caudal pattern of pathology within the corpus callosum in the cuprizone mouse model. J. Magn. Reson. Imaging 27, 446-453.

Xie, M., Tobin, J., Buddle, M., Chen, C., Trinkaus, K., Cross, A., et al., 2010. Rostrocaudal analysis of corpus callosum demyelination and axon damage across disease stages refines diffusion tensor imaging correlations with pathological features. J. Neuropathol. Exp. Neurol. 69, 704-716. 